\title{
Investigation of Li metal plating and dissolution on Graphite Electrodes
}

Simon Hein ${ }^{1,2}$, Christin Hogref ${ }^{3}$, Thomas Waldmann ${ }^{3}$, Timo Danner ${ }^{1,2}$, Karten Richter ${ }^{3}$, Margret WohlfahrtMehrens ${ }^{2,3}$, Arnulf Latz ${ }^{1,2,4}$

${ }^{1}$ Department of Computational Electrochemistry, Institute of Engineering Thermodynamics, German Aerospace Centre (DLR), Pfaffenwaldring 38-40, 70569 Stuttgart, Germany,

${ }^{2}$ Helmholtz-Institute Ulm for Electrochemical Energy Storage, Helmholtzstrasse 11, 89081 Ulm, Germany

${ }^{3}$ Zentrum für Sonnenenergie- und Wasserstoff-Forschung Baden-Württemberg, Helmholtzstrasse 8, 89081 Ulm, Germany

${ }^{4}$ Institute of Electrochemistry, University Ulm, Albert-Einstein-Allee 11, 89069 Ulm, Germany

Email: Simon.Hein@dlr.de

Li plating, meaning the deposition of metallic Lithium on the negative electrode is considered as one of the major degradation mechanisms in Li-Ion batteries. Therefore, understanding the processes which occur between metallic Lithium and state-of-the-art electrode materials, mainly Graphite, is important to improve the life-time of Li ion cells.

Lithium plating and stripping is explored using an electrochemical model, which explicitly includes the growth of plated lithium as a competing process to the direct intercalation lithium ions [1]. The model is combined with a thermodynamically consistent transport theory and implemented in the simulation framework Battery and Electrochemistry Simulation Tool (BEST). The extended framework is used to conduct a first study regarding the impact of an inhomogeneous SEI growth on the deposition of metallic lithium.

Moreover, we will present recent work on simulations of model experiments investigating the dissolution of lithium deposits on Graphite electrodes [2]. Different electrolyte formulations and configurations of a lithium metal disk on the electrode are investigated to evaluate different lithium transport pathways. In each experiment some pathways are intentionally blocked, thus yielding interesting insights into the dissolution mechanism. Postmortem analysis on the Graphite electrodes is performed using Raman spectroscopy and glow-discharge optical emission spectroscopy (GD-OES) revealing the spatial distribution of the lithium at the end of the dissolution experiment. At the same time measurements of the open circuit voltage are performed to monitor the progress of the dissolution process. Our simulations are in very good qualitative agreement with measured OCV curves and the observed lithium distributions.

Therefore, the simulations provide additional "in-operando" insights to the plating as well as the dissolution of a metallic lithium phase. With the mechanistic insights optimization of the electrode and cell architecture can be deduced which help to improve the life-time of Li ion cells.

[1] S. Hein, T. Danner, and A. Latz, "An electrochemical model of lithium plating and stripping in lithium-ion batteries," ACS Appl. Energy Mater., vol. 3, no. 9, p. acsaem.0c01155, Jul. 2020.

[2] C. Hogrefe et al., "Mechanistic Details of the spontaneous Intercalation of Li Metal into Graphite Electrodes," J. Electrochem. Soc., Nov. 2020. 